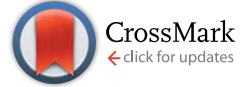

Cite this: RSC Adv., 2017, 7, 4671

Received 3rd October 2016 Accepted 28th November 2016

DOI: $10.1039 / c 6 r a 24682 f$

www.rsc.org/advances

\section{Polycyclic aromatic hydrocarbons in traditional Chinese medicines: an analytical method based on different medicinal parts, levels, distribution, and sources $\uparrow$}

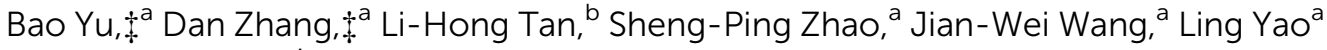 \\ and Wei-Guo Cao*ac
}

Herein, we describe a rapid, easy, and cost-effective high-performance liquid chromatography method using UV and fluorescence detectors for the simultaneous analysis of 16 polycyclic aromatic hydrocarbons (PAHs) in traditional Chinese medicines (TCMs). Pretreatment involved different extraction methods depending on the different medicinal parts, and was followed by silica gel purification. The method was validated and used to assess PAHs contamination in 32 TCMs. In the samples analyzed, all 16 PAHs were present. Their total contents ranged from 19.5 to $1614.1 \mu \mathrm{g} \mathrm{kg}^{-1}$. Among all PAHs studied, phenanthrene was the most common and serious contaminant, followed by fluorene and fluoranthene. Leaves had the highest levels of the 16 PAHs, followed by roots and stems, seeds, flowers, and fruits. The diagnostic ratios and principle component analysis showed that the main sources of PAHs in TCMs were both pyrogenic and petrogenic. Furthermore, PAHs in roots and stems primarily originated from wood or coal combustion, as reported for the first time. Our results suggest that PAHs contamination in TCMs is widespread, and that the proposed method may be a useful tool for quality control of PAHs in TCMs, and for determining their potential health risks.

\section{Introduction}

Polycyclic aromatic hydrocarbons (PAHs), a class of over 200 different compounds containing two or more fused aromatic rings, are primarily formed by incomplete combustion or heatinduced decomposition of organic matter. ${ }^{1}$ They may interfere with the normal function of $\mathrm{DNA}^{2}$ and some have been proven highly carcinogenic, mutagenic, and genotoxic in experiments on animals. ${ }^{3}$ PAHs are widely present in the environment due to their hydrophobic properties, allowing adsorption onto atmospheric particles and direct deposition in sediments, soils, and plants. ${ }^{4}$ Traditional Chinese Medicines (TCMs), are largely herbal in nature and are susceptible to contamination with PAHs. Besides environmental pollution, different forms of processing undergone by TCMs, such as drying, can also lead to the PAH

${ }^{a}$ College of Traditional Chinese Medicine, Chongqing Medical University, Chongqing, China

${ }^{b}$ Department of Pharmaceutics, Chongqing Medical and Pharmaceutical College, Chongqing, China

'The Laboratory of Traditional Chinese Medicine, Chongqing Medical University, Chongqing, China. E-mail: cwgzd2001@hotmail.com

$\dagger$ Electronic supplementary information (ESI) available. See DOI: 10.1039/c6ra24682f

‡ Bao Yu and Dan Zhang contributed equally to this work; they are co-first authors. contamination. ${ }^{5}$ Due to their specific theory and long historical clinical practice, TCMs are gaining increasing attention; an estimated 1.5 billion people now use them worldwide. ${ }^{6}$ Therefore, it is important to monitor PAHs levels to evaluate the potential risks associated with human consumption of TCMs. The following 16 PAHs have been listed as priority organic pollutants by the United States Environmental Protection Agency (USEPA): acenaphthylene (ACL), anthracene (AN), acenaphthene (AC), benzo $[a]$ pyrene $(\mathrm{BaP})$, benzo[a]anthracene $(\mathrm{BaA})$, benzo $[b]$ fluoranthene (BbFA), benzo[ $k]$ fluoranthene (BkFA), benzo $[g, h, i]$ perylene (BghiP), chrysene (CHR), dibenzo[ $a, h]$ anthracene (DBahA), fluoranthene (FA), fluorene (FL), indeno[1,2,3-c,d]pyrene (IP), naphthalene (NA), phenanthrene (PHE), and pyrene (PY).

Various analytical methods can be used to quantify PAHs, of which high-performance liquid chromatography, coupled with fluorescence (HPLC-FLD) or ultraviolet (UV)-visible detection, , ${ }^{5,7}$ as well as gas chromatography-tandem mass spectrometry (GC-MS/MS $)^{9,10}$ are the two most common analytical techniques applied in recent years. The advantage of using UV and fluorescence detections in series with HPLC is that UV detection is required for acenaphthylene because it is inactive to fluorescence. Owing to the complex matrix of samples to be analyzed, optimization of the extraction and cleanup procedures is indispensable. Until now, pretreatment for PAHs in a matrix has commonly relied on a two-stage methodology, involving liquid- 
liquid extraction with various solvents (acetone, acetonitrile, hexane, cyclohexane, and methylene chloride) and a solid-phase extraction (SPE) cleanup using alumina, florisil, silica, and $\mathrm{C}_{18}$ cartridges, or gel permeation chromatography (GPC). ${ }^{\mathbf{1 0 - 1 4}} \mathrm{A}$ modified QuEChERS method has also been reported by Magdalena Surma et al. ${ }^{15}$ which provided a significant reference method and guidance for the analysis of PAHs in TCMs.

However, TCMs have many different medicinal parts, including roots, stems, flowers, fruits, seeds, and leaves. Different medicinal parts have different types of matrices; therefore, different pretreatments should be established for different sample types. To the best of our knowledge, there had only been one study on PAHs determination in different parts of TCMs, ${ }^{9}$ wherein purification procedures were conducted using different SPE columns, which was complicated and costly. Therefore, this study aimed to develop and validate a simple and easy method for determining the levels of 16 EPA PAHs in TCMs based on medicinal parts. The pretreatment consisted of three different extraction methods-ultrasonic extraction, homogenization extraction, and oscillation extraction-followed by a silica gel cleanup. Subsequently, analysis was carried out using HPLC coupled to UV and fluorescence detectors in series, which ensured the detection of all 16 PAHs. In addition, we further discussed the distribution and source apportionment of PAHs in different types of TCMs, which has not been reported previously. This work could provide more information to reduce PAHs in TCMs from the source.

\section{Experimental}

\subsection{Material and reagents}

Thirty-two TCM samples, including six roots and stems, eight fruits, eight seeds, five flowers, and five leaves, were purchased from local market. They were manufactured at the place of origin and kept in cool and dry conditions. The TCMs were powdered, passed through a $0.5 \mathrm{~mm}$ metal sieve, and stored at $4{ }^{\circ} \mathrm{C}$ until analysis.

All glassware was cleaned with detergent, followed by ultrapure water, and finally rinsed with solvents and dried in a hot air oven. A standard 16 PAHs mixture in benzene/methylene chloride solution $(1: 1, \mathrm{v} / \mathrm{v})$ containing $2000 \mu \mathrm{g} \mathrm{mL} \mathrm{mL}^{-1}$ of each component was purchased from Aladdin Co. (CA, USA). Hexane, acetone, acetonitrile, and methylene chloride were all HPLC grade and obtained from Sigma-Aldrich (St. Louis, MO, USA). Anhydrous sodium sulfate (Merck, India) was cleaned with solvents in a Soxhlet apparatus, dried at $110^{\circ} \mathrm{C}$ for $3 \mathrm{~h}$ and stored in a sealed desiccator before use. Silica gel (100-200 mesh) was purchased from Supelco (Sigma-Aldrich, USA) and activated at $105{ }^{\circ} \mathrm{C}$ for $2 \mathrm{~h}$ before use. SPE columns (ProElut $\mathrm{C}_{18}, 1 \mathrm{~g} / 6 \mathrm{~mL}$ ) used for purification were obtained from Dikma Technologies (Beijing, China). Ultra-high-quality water was produced by a Milli-Q water purification system (Millipore, Madrid, Spain).

\subsection{Sample pretreatment}

2.2.1. Extraction. The extraction strategy was optimized and established according to the different medicinal parts of
TCMs. For roots and stems, the sample powder (1 g) and methylene chloride $(20 \mathrm{~mL})$ were added to a $50 \mathrm{~mL}$ conical flask. The flask was placed in an ultrasonic bath operating at a frequency of $70 \mathrm{kHz}$ for $20 \mathrm{~min}$. For leaves and flowers, the sample $(1 \mathrm{~g})$ and methylene chloride $(20 \mathrm{~mL})$ were transferred into a glass centrifuge tube, and extraction was performed by homogenization at $3000 \mathrm{rpm}$ for $3 \mathrm{~min}$. For fruits, the sample $(1 \mathrm{~g})$ was extracted with hexane $(20 \mathrm{~mL})$ by shaking for $60 \mathrm{~min}$ at $1500 \mathrm{rpm}$. For seeds, the sample $(1 \mathrm{~g})$ and acetonitrile/acetone $(20 \mathrm{~mL} ; 3: 2, \mathrm{v} / \mathrm{v})$ were mixed in a conical flask and sonicated for $20 \mathrm{~min}$ at $70 \mathrm{kHz}$. All the mixtures above were subsequently centrifuged at $3000 \mathrm{rpm}$ for $5 \mathrm{~min}$, and the supernatants decanted and collected. The residues were extracted again with another $20 \mathrm{~mL}$ of their corresponding extraction solvent. The combined supernatants were concentrated by a rotary evaporator at $35{ }^{\circ} \mathrm{C}$ to around $1 \mathrm{~mL}$ for further purification.

2.2.2. Purification. Purification procedures also varied with different medicinal parts. For roots and stems, fruits, leaves, and flowers, the concentrated supernatant was purified by column chromatography with silica gel using a glass column $(300 \times 15 \mathrm{~mm}$ i.d. $)$ packed with $3 \mathrm{~g}$ activated silica gel and $1 \mathrm{~g}$ anhydrous sodium sulfate at the top. The eluent was collected after the extract was applied to the top of the column, and the column was washed with $20 \mathrm{~mL}$ of a mixture of hexane and methylene chloride $(1: 1, \mathrm{v} / \mathrm{v})$. Both eluents were combined and evaporated to near dryness in a rotary evaporator at $35^{\circ} \mathrm{C}$ under vacuum. Seed extracts were cleaned up using $\mathrm{C}_{18}$ columns before purification on silica gel. About $1 \mathrm{~mL}$ of the seeds extract was directly transferred onto a $\mathrm{C}_{18}$ column pre-activated with $4 \mathrm{~mL}$ methanol and acetonitrile and eluted twice with $8 \mathrm{~mL}$ acetonitrile/acetone $(3: 2, \mathrm{v} / \mathrm{v})$. The eluent was collected and the solvent was changed into methylene chloride, before further cleaning up on a homemade silica gel column, as described above. After removing solvents, the residue obtained was dissolved in $1 \mathrm{~mL}$ of acetonitrile and filtered through $0.45 \mu \mathrm{m}$ polytetrafluoroethylene (PTFE) filters, and injected for HPLCUV-FLD analysis.

\subsection{PAHs analysis}

PAHs analysis was carried out using a Shimadzu HPLC apparatus equipped, in series, with a LC-20AD pump, SPD-20A UVVis detector, and RF-20A fluorescence detector. Chromatographic separation was performed on a LC-PAH Supelcosil ${ }^{\mathrm{TM}}$, an analytical column $(25 \mathrm{~cm} \times 4.6 \mathrm{~mm}, 5 \mu \mathrm{m}$ film $)$ using a $20 \mu \mathrm{L}$ sample injection volume and a gradient flow using acetonitrile (A) and water (B) at $1.0 \mathrm{~mL} \mathrm{~min}^{-1}$. The gradient elution program was as follows: $0 \rightarrow 6 \mathrm{~min}$, isocratic $56 \% \mathrm{~A} ; 6 \rightarrow 17 \mathrm{~min}$, linear gradient 56\% A $\rightarrow$ 88\% A; $17 \rightarrow 38$ min, linear gradient 88\% A $\rightarrow 86 \% \mathrm{~A} ; 38 \rightarrow 39 \mathrm{~min}$, linear gradient $86 \% \mathrm{~A} \rightarrow 56 \% \mathrm{~A} ; 39 \rightarrow$ $45 \mathrm{~min}$, isocratic $56 \% \mathrm{~A}$. The temperature in the column oven was set to $35{ }^{\circ} \mathrm{C}$. All 16 PAHs were determined using a UV-Vis detector set at $229 \mathrm{~nm}$ and the fluorescence detector with variations in the wavelength excitement (Ex) and emission (Em) as follows: (1) NA - Ex/Em 270/324, (2) AC - Ex/Em 270/324, (3) FL Ex/Em 270/324, (4) PHE - Ex/Em 248/375, (5) AN - Ex/Em 248/ 375, (6) FA - Ex/Em 280/462, (7) PY - Ex/Em 270/385, (8) BaA - 
Ex/Em 270/385, (9) CHR - Ex/Em 270/385, (10) BbFA - Ex/Em 256/446, (11) BkFA - Ex/Em 292/410, (12) BaP - Ex/Em 292/ 410, (13) DBahA - Ex/Em 292/410, (14) BghiP - Ex/Em 292/410, and (15) IP - Ex/Em 302/507. Before analyzing a sample set, blank solvent was injected to insure that the system was free of contaminants or interfering peaks.

\subsection{Qualitation and quantification}

The qualitation of PAHs in TCMs was achieved by comparing their retention times with those of standard PAHs. The external standard plot method was used for quantification. The stock solution containing $2000 \mu \mathrm{g} \mathrm{mL} \mathrm{m}^{-1}$ of all 16 PAHs was diluted successively in acetonitrile to construct an analytical curve, and each standard solution was prepared in duplicate.

The limits of detection (LOD) and quantification (LOQ) of the method were calculated from the signal-to-noise $(\mathrm{S} / \mathrm{N})$ ratio of standard solutions. The LOD corresponded to the amount of analyte for which the $\mathrm{S} / \mathrm{N}$ ratio of the peak area was equal to 3 , while the LOQ corresponded to an S/N ratio of 10.

\subsection{Recovery study}

Recovery experiments were performed by spiking four representative samples (Rhizoma dioscoreae, Mulberry leaves, Fructus mume, and Fructus cannabis) from four TCM categories with three different concentrations of the standard PAHs solution $\left(10,50\right.$, and $\left.100 \mu \mathrm{g} \mathrm{kg}^{-1}\right)$. To ensure the proposed method was accurate for every TCM selected, the other selected TCMs were also spiked with $50 \mu \mathrm{g} \mathrm{kg}^{-1}$ of the standard solution to conduct the recovery study. The spiked samples and the unspiked controls were analyzed in triplicate. Recoveries were calculated from the differences in total amounts of each PAH between the spiked and unspiked samples.

The repeatability was estimated for all PAHs during the recovery studies and expressed as the relative standard deviation $(n=3)$. The intra-day precision was determined by analysing the same standard mixture $\left(100 \mu \mathrm{g} \mathrm{kg}^{-1}\right.$ for each PAH $)$ six times on the same day with the same instrument and the same operator, while the inter-day precision was calculated on the basis of the results from two different days and from the different operators. The result was also expressed as the relative standard deviation.

\subsection{Source apportionment}

Ratios of individual PAHs, such as the diagnostic ratios of $\mathrm{AN} /$ $(\mathrm{AN}+\mathrm{PHE}), \mathrm{FA} /(\mathrm{FA}+\mathrm{PY})$, and $\mathrm{BaA} /(\mathrm{BaA}+\mathrm{CHR})$, are frequently utilized as diagnostic tools to distinguish the $\mathrm{PAH}$ sources. $^{16-18}$

Moreover, principal component analysis (PCA) can be used to analyze the sources of PAHs. PCA is known as a dimensional reduction because the method is able to decrease the dimensionality of the primary set of data (measured PAH contents in TCM samples) and compress data into a lower dimensional matrix (principal components). ${ }^{19}$ By utilizing the orthogonal transformation method, principle components (PCs) were extracted with different factor loadings indicating correlations between each pollutant species and each PC. ${ }^{\mathbf{1 6}, 20}$ Each PC was further evaluated and recognized by source markers or profiles as reasonable pollution sources.

\section{Results and discussion}

\subsection{Optimization of sample pretreatment}

3.1.1. Extraction. TCMs have complex matrices comprising a variety of chemical components, and different parts of medicinal plants have different matrix categories. In other words, the same medicinal parts have similar matrices. For example, there are numerous pigments in almost all leaves and flowers, most seeds are full of fats, and carbohydrates might be the main interferences in fruit herbal medicines. Considering the large number of TCMs, it is necessary and feasible to establish a representative matched pretreatment for each category of TCMs.

Four representative TCMs (Rhizoma dioscoreae, Mulberry leaves, Fructus mume, and Fructus cannabis) were chosen from four categories of TCM medicinal parts and used to conduct the optimization of extraction methods, including ultrasonic extraction, homogenization extraction, and oscillation extraction. The total yields of 16 PAHs ( $\Sigma 16$ PAHs) and their average recoveries (by spiking $50 \mu \mathrm{g} \mathrm{kg}^{-1}$ for each $\mathrm{PAH}$ ) were adopted to evaluate the methods. The optimization results are shown in Table S1.† For seeds, roots, and stems, it was observed that ultrasonic extraction had the highest total yield and average recovery, while oscillation and homogenization extraction were more suitable for fruits and leaves/flowers, respectively.

The solvents used to extract PAHs from plant samples were usually hexane, acetone, methylene chloride, acetonitrile, or a mixture thereof. Weak polar solvents such as hexane and methylene chloride were used in the experiment owing to the low polarity of PAHs and less polar interferences. However, for seeds, polar solvents acetonitrile and acetone were used, in order to reduce the extraction of fats in the sample and coordinate with subsequent $\mathrm{C}_{18}$ purification without exchanging solvents. Different parts of medicinal plants have different matrix categories, so different solvent systems should be used and optimized to ensure full extraction and high recoveries. For seeds, three solvents, including acetone, acetonitrile, and acetonitrile/acetone $(3: 2, \mathrm{v} / \mathrm{v})$, were compared using the representative sample, Fructus cannabis. For the other groups, hexane, methylene chloride, and hexane/methylene chloride $(1: 1, \mathrm{v} / \mathrm{v}$ ) were investigated to seek proper solvents (see Table $\mathrm{S} 2 \dagger)$. In addition, different extraction times were also studied for every extraction method (see Table S3†). The final established extraction conditions were as described above.

3.1.2. Purification. All purification processes were conducted directly by silica gel column, except for seeds, which were first cleaned up using a $\mathrm{C}_{18}$ column. Injection of unclean sample extracts resulted in the generation of substantial backpressure in the analytical column, reduced retention reproducibility, and interfered with peak identification. ${ }^{21}$ The problem disappeared when silica gel cleanup was used, because silica gel had a strong affinity for polar pigments and other polar interferences. Considering the non-polar, lipophilic nature of PAHs, silica gel was enough to complete the 
purification process, which was easier and less costly than other reports., ${ }^{9,10}$ The recoveries of the sixteen individual PAHs ranged from $66.7 \%$ to $108.2 \%$ (Tables 2 and $S 4 \dagger$ ), which ensured the accuracy and feasibility of the pretreatment. Furthermore, silica gel purification was reported to be low-cost, effective, and easy for many researchers to perform for $\mathrm{PAH}$ quantification in environmental and human health-risk assessments. ${ }^{22-24}$ For seeds, the silica gel cleanup was not capable of removing coextracted fats, so a $\mathrm{C}_{18}$ column, which could retain the nonpolar or weak polar pigments or fats in sample extracts, was used before silica gel to remove fats.

Standard solution $\left(1 \mathrm{~mL}, 200 \mathrm{ng} \mathrm{mL}{ }^{-1}\right)$ was transferred to pre-activated silica gel and $\mathrm{C}_{18}$ columns to conduct optimization experiments on choosing the proper eluent and eluent volume by comparing the total yield of 16 PAHs. As shown in Fig. $\mathrm{S} 1$ and $\mathrm{S} 2, \uparrow$ hexane/methylene chloride $(20 \mathrm{~mL} ; 1: 1, \mathrm{v} / \mathrm{v})$ was a suitable eluent for silica gel and was enough for $8 \mathrm{~mL}$ acetonitrile/acetone $(3: 2, \mathrm{v} / \mathrm{v})$ to wash away all PAHs attracted to the $\mathrm{C}_{18}$ column.

\subsection{Method validation}

HPLC elution conditions were optimized to ensure the good separation of all 16 PAHs; the chromatogram is shown in Fig. 1. In order to detect all 16 PAHs and obtain the best detection limit, fluorescence and UV detectors were connected in series. ACL was inactive in fluorescence and detected by UV at $229 \mathrm{~nm}$, whereas the other 15 PAHs were detected by fluorescence detector.

The calibration curve was obtained by regression of the peak area with standard solution concentration. As shown in Table 1, all calibration curves were highly linear (with correlation coefficient $R^{2} \geq 0.9994$ ) in the range of concentration examined.

The interval for the limit of detection (LOD) for all 16 PAHs was from 0.015 to $0.300 \mu \mathrm{g} \mathrm{kg}^{-1}$, while the limit of quantification (LOQ) ranged from 0.050 to $1.000 \mu \mathrm{g} \mathrm{kg}{ }^{-1}$. All LODs were below $0.030 \mu \mathrm{g} \mathrm{kg}^{-1}$, except for ACL $\left(0.300 \mu \mathrm{g} \mathrm{kg}^{-1}\right)$, which was 10 times higher than the other PAHs, explained by the UV sensitivity being much lower than FLD. Compared with LOD values from previous reports, ${ }^{\mathbf{9 , 1 0 , 2 5}}$ it was clear that the proposed method had sufficient sensitivity for the determination of PAHs in TCMs.

Recovery experiments were carried out by adding 10,50 , and $100 \mu \mathrm{g} \mathrm{kg}{ }^{-1}$ of each analyte standard to four representative samples. The results are shown in Table 2. Overall, the spiked recoveries ranged from 66.7 to $97.5 \%$ for all $\mathrm{PAHs}$, indicating that the method accuracy was satisfactory. Moreover, to ensure the method was accurate for every sample tested in this study, the other TCMs selected were also spiked with $50 \mu \mathrm{g} \mathrm{kg}$ standard solution to conduct recovery studies. The results, presented in Table $\mathrm{S} 4, \uparrow$ ranged from 69.5 to $108.2 \%$, confirming good method accuracy for all TCM samples involved in the study. The relative standard deviations (RSDs) did not exceed $10 \%$ in any instance. The repeatability, and intra and inter-day precision results are also shown in Table 2; all were below $8 \%$ (RSD), demonstrating the high repeatability and precision of the method.

\subsection{Application to real samples}

After validation, the proposed method was applied to evaluate the 16 PAHs contamination in 32 TCMs, including six roots and stems, eight fruits, eight seeds, five flowers, and five leaves.

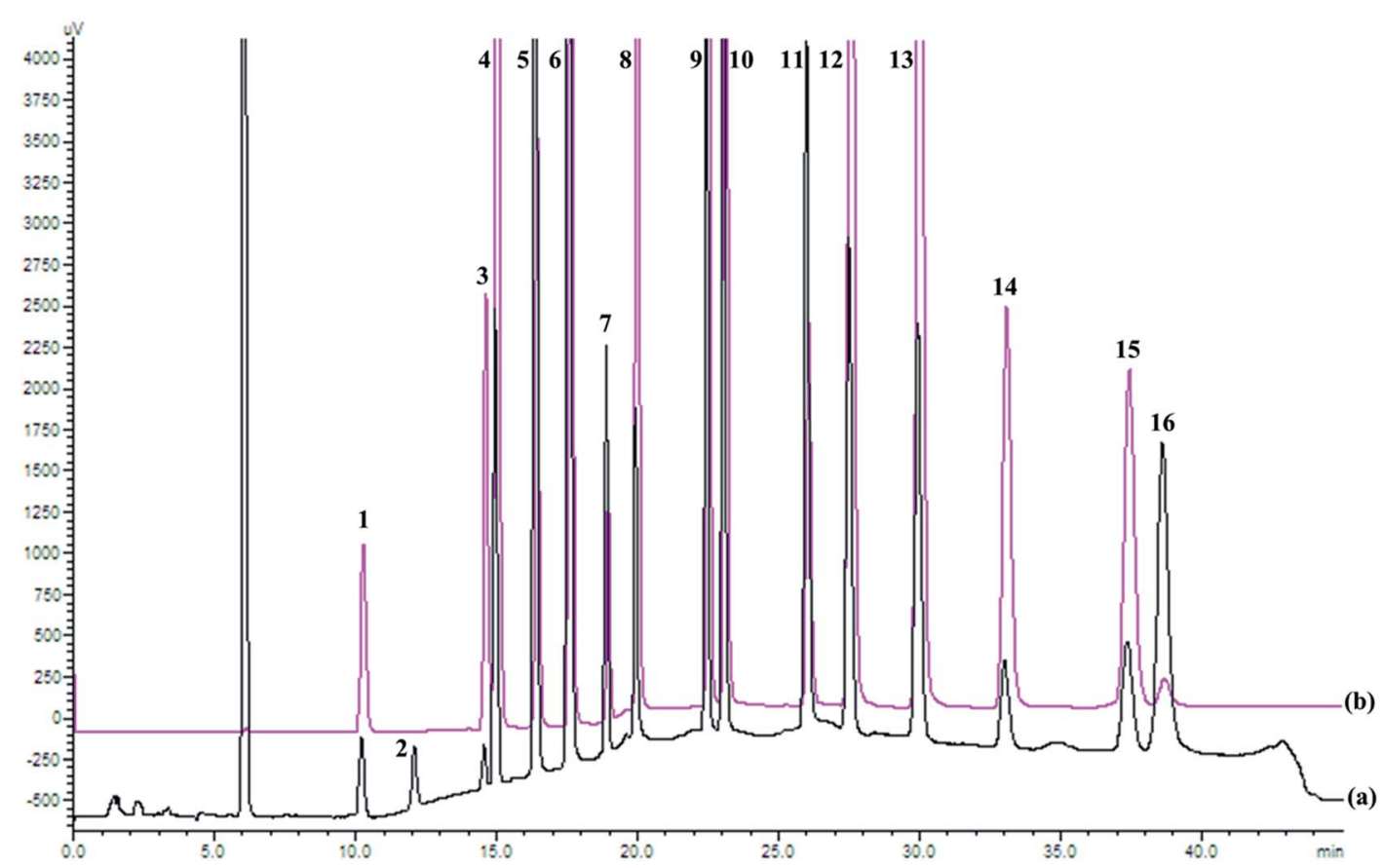

Fig. 1 Chromatogram of the standard solution monitored by both (a) UV and (b) fluorescence detection (peaks identification: 1, NA; 2, $A C L ; 3$, AC; 4, FL; 5, PHE; 6, AN; 7, FA; 8, PY; 9, BaA; 10, CHR; 11, BbFA; 12, BkFA; 13, BaP; 14, IP; 15, DBahA; and 16, BghiP). 
Table 1 Analytical performance of the proposed method

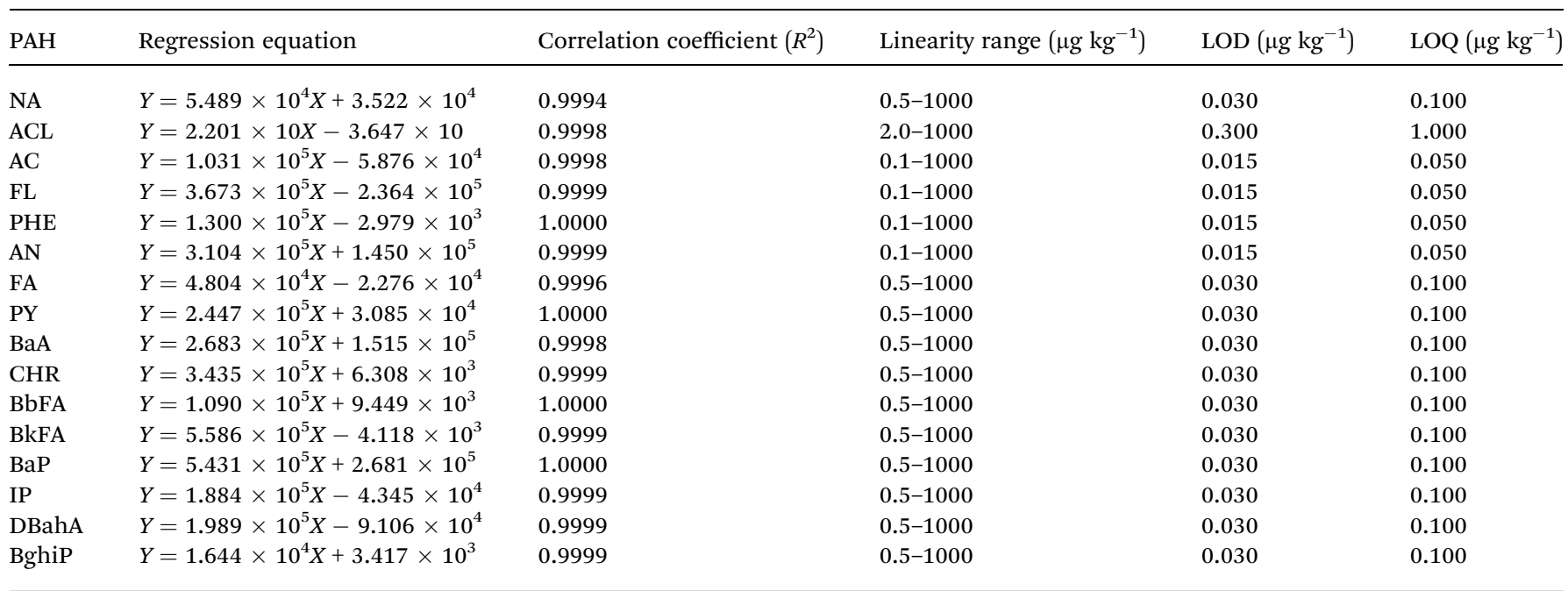

Table $55 \uparrow$ shows the results obtained, expressed as the mean of three replications \pm standard deviation $\left(\mu \mathrm{g} \mathrm{kg}{ }^{-1}\right)$. Concentrations below the limit of detection were considered as not detected (nd).

All 32 TCMs were contaminated with some PAHs, but with a large variability in each $\mathrm{PAH}$ level in different samples, which might be attributed to different $\mathrm{PAH}$ sources for each sample. The total levels of the 16 PAHs varied from $19.5 \mu \mathrm{g} \mathrm{kg}{ }^{-1}$ (Fructus rubi) to $1614.1 \mu \mathrm{g} \mathrm{kg} \mathrm{kg}^{-1}$ (Radix liquiritiae), with an average of $376.8 \mu \mathrm{g}$ $\mathrm{kg}^{-1}$. Among all PAHs studied, PHE was the most common and serious contaminant (found in 32 of 32 samples; 100\%), followed by FL (31 samples; 97\%) and FA (31 samples; 97\%). Similar results were found for PHE and FA in some fruit and herbal teas, ${ }^{\mathbf{2 6}}$ for PHE, FA, ACL, FL and PY in 24 Chinese herbal medicines, ${ }^{9}$ and for PY, FA and NA in tea products and crude drugs. ${ }^{5}$ Furthermore, BghiP was detected only in Radix liquiritiae $\left(4.8 \mu \mathrm{g} \mathrm{kg}^{-1}\right)$, which had the lowest detection rates among the 16 PAHs.

The highest concentration of any $\mathrm{PAH}$ in the studied medicinal plants was that of PHE in Radix liquiritiae $\left(586.4 \mu \mathrm{g} \mathrm{kg}^{-1}\right)$. Radix liquiritiae was also the only TCM in which all 16 PAHs were detected, the total content of PAHs reaching $1614.1 \mu \mathrm{g} \mathrm{kg}{ }^{-1}$. Cui et al. ${ }^{9}$ reported a similar result of $1842.8 \mu \mathrm{g} \mathrm{kg}{ }^{-1}$, indicating that Radix liquiritiae may be vulnerable to $\mathrm{PAH}$ contaminants and that special attention should be paid to improve its quality. In particular, $\mathrm{BaP}$, one of the most potent carcinogenic PAHs, was detected in 8 of 32 samples. Its contents in 8 samples ranged from 10.3 to $32.8 \mu \mathrm{g} \mathrm{kg}^{-1}$, all of which exceeded levels set for BaP by EU regulations for foods $\left(2 \mu \mathrm{g} \mathrm{kg}^{-1}\right)$.

\subsection{Distribution analysis}

Fig. 2 shows the distribution of mean values for the sums of 16 PAHs ( $\Sigma 16$ PAHs), low molecular weight PAHs ( $\Sigma$ L-PAHs), and high molecular weight PAHs ( $\Sigma$ H-PAHs), as well as PAHs with the highest levels in the five medicinal parts of TCMs. Among the different medicinal parts, the highest level of $\Sigma 16$ PAHs was found in leaves $\left(747.0 \mu \mathrm{g} \mathrm{kg}^{-1}\right)$, followed by roots and stems $\left(649.6 \mu \mathrm{g} \mathrm{kg}{ }^{-1}\right)$, seeds $\left(255.7 \mu \mathrm{g} \mathrm{kg}^{-1}\right)$, flowers $\left(233.3 \mu \mathrm{g} \mathrm{kg}^{-1}\right)$, and, finally, fruits (151.7 $\left.\mu \mathrm{g} \mathrm{kg}^{-1}\right)$, which was consistent with the order for levels of $\Sigma$ L-PAHs. However, for $\Sigma$ H-PAHs, the highest levels were found in roots and stems, followed sequentially by leaves, seeds, flowers, and fruits. Moreover, the highest levels found were for PHE in leaves $\left(236.8 \mu \mathrm{g} \mathrm{kg}^{-1}\right)$, seeds $\left(50.3 \mu \mathrm{g} \mathrm{kg}^{-1}\right)$, roots and stems $\left(180.0 \mu \mathrm{g} \mathrm{kg}^{-1}\right)$, for NA in fruits $\left(45.1 \mu \mathrm{g} \mathrm{kg}^{-1}\right)$, and for ACL in flowers $\left(45.7 \mu \mathrm{g} \mathrm{kg}^{-1}\right)$.

Leaves had the highest levels of $\Sigma 16$ PAHs and $\Sigma$ L-PAHs among medicinal parts, which might be related to leaves taking longer time to grow and having a greater surface area than other parts, thus resulting in a longer exposure to PAHs and higher accumulation of PAHs. ${ }^{25,27}$ Flowers also have a high surface area, but the contamination was not as serious as in leaves due to their shorter growth cycle. Compared with fruits, the seeds, most of which were rich in fat, had higher levels of $\Sigma 16$ PAHs, $\Sigma$ L-PAHs, and $\Sigma$ H-PAHs. This could be explained by the lipophilic compounds contributing to the accumulation of hydrophobic PAHs in seeds and, consequently, causing a higher PAH contamination level. ${ }^{26}$ Roots and stems had the highest level of $\Sigma$ H-PAHs and the second highest level of $\Sigma 16 \mathrm{PAHs}$; possible reasons for this are soil-to-root transfer and atmosphere-to-plant pathway, ${ }^{27}$ or the different forms of processing to which the samples were submitted, such as the drying process. This will be discussed further in the next section ("Source analysis").

From Table S5† and Fig. 2, it was obvious to see that low molecular weight PAHs, accounting for $45.8-94.9 \%$ of total PAHs with a mean value of $77.0 \%$, predominated over high molecular weight PAHs in all TCM groups. The percentage of heavy PAHs, for which genotoxic, mutagenic, and carcinogenic properties have been stated, among all PAHs was generally low. Similar results have also been observed in the literature for tea samples, ${ }^{13}$ fruit and herbal teas samples, ${ }^{26}$ and in tea and coffee samples. ${ }^{28}$

\subsection{Source analysis}

PAHs originate from both the natural as well as anthropogenic sources, and anthropogenic sources were the main ones. 


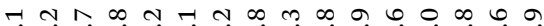

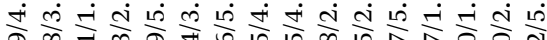

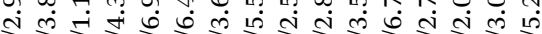

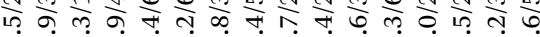

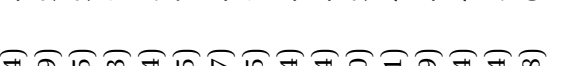

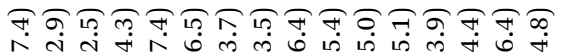

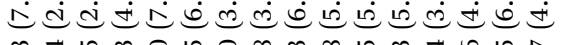

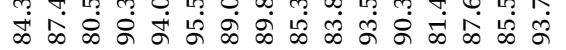

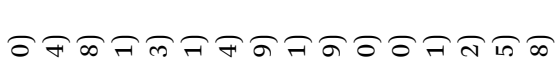

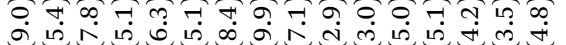

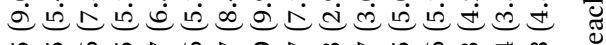
10 10

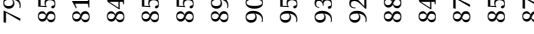
เฺ

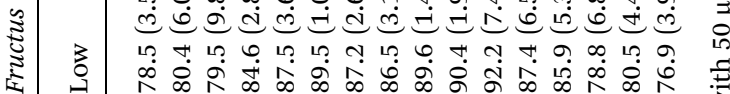
10 โอำ

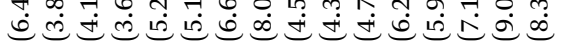

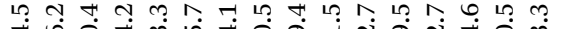

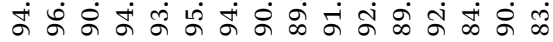

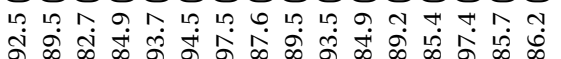

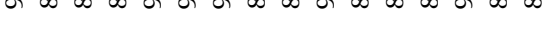

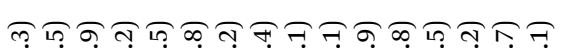

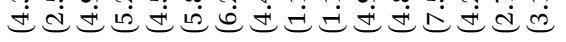

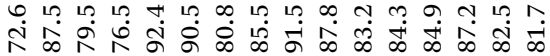

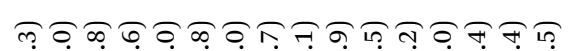

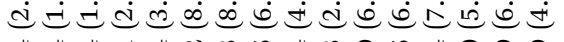
ᄀ. ம்

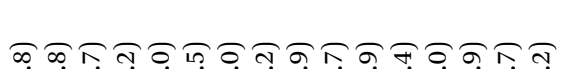

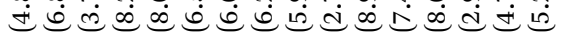

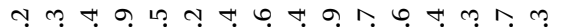

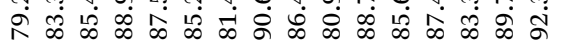

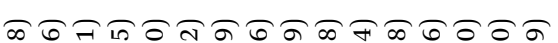

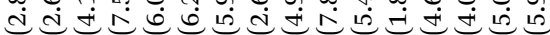

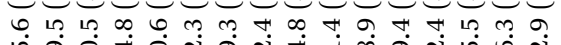
ம்

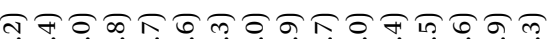

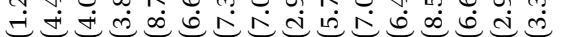
耐 2000

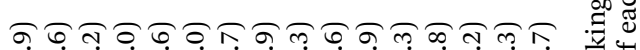

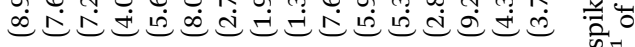
$\infty$ \# 0 .

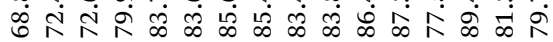

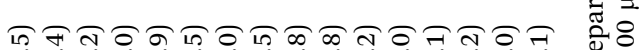

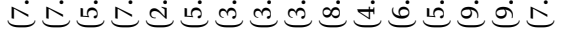

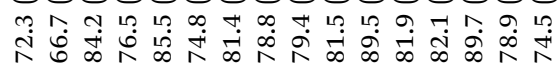

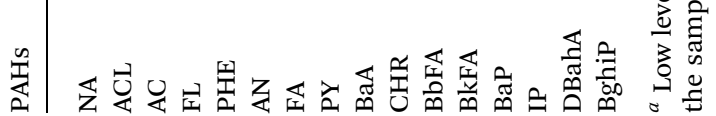




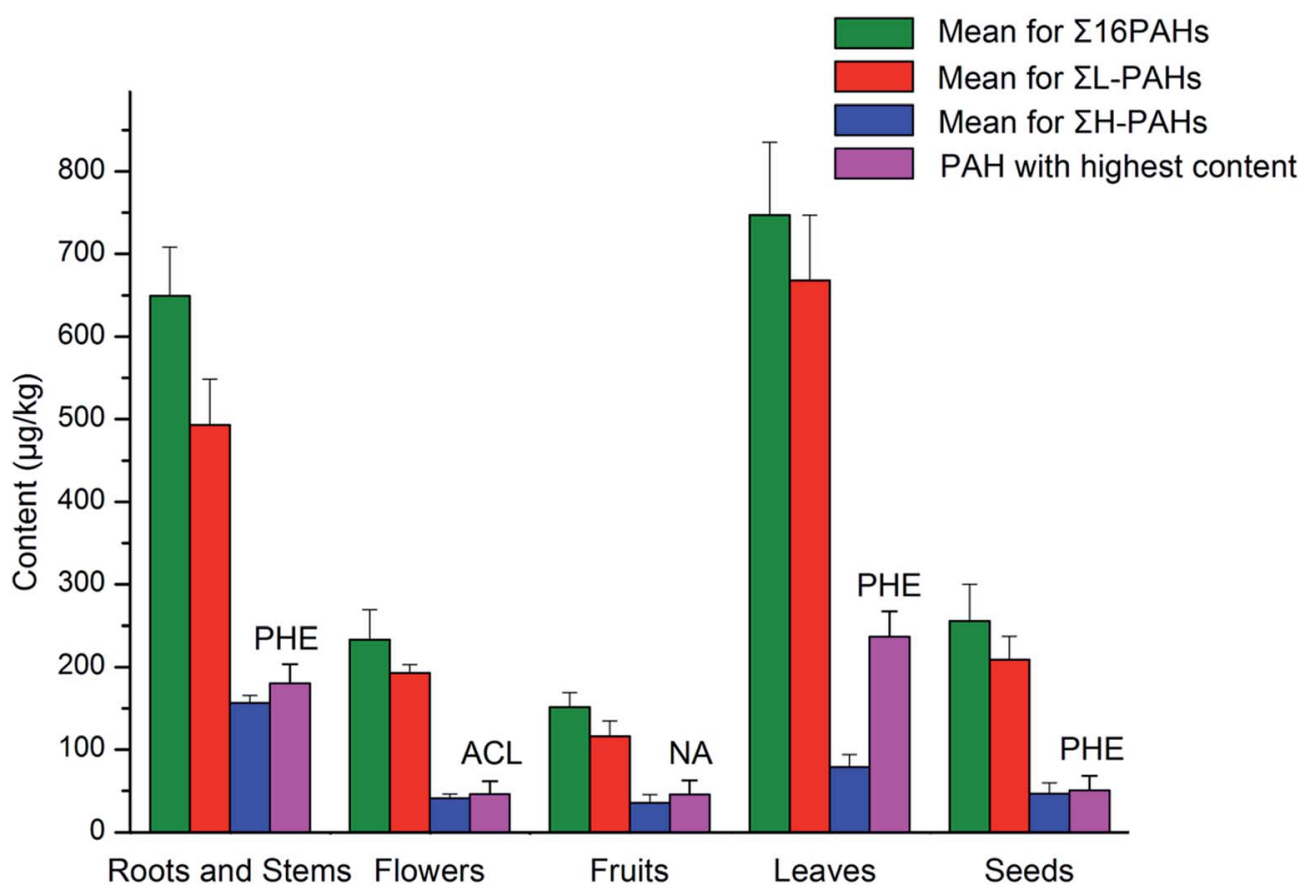

Fig. 2 Distribution of total levels of $16 \mathrm{PAHs}$, light PAH levels, and heavy PAH levels, as well as PAHs with the highest contents in the different medicinal parts of TCMs.

Anthropogenic sources of PAHs can be categorized into pyrogenic and petrogenic groups. ${ }^{29}$ With pyrogenic sources, PAHs are generated by combustion of fossil fuel (coal and petroleum) and biomass. The petrogenic origin was from discharge of crude oil and petroleum products. ${ }^{30}$ This research focuses on the ratio method to conduct qualitative analysis of the sources of PAHs.

3.5.1. Diagnostic ratio analysis. Concentration ratios of some selected PAHs are considered characteristic of their sources and have widely been used to infer their source nature. Yunker et al. ${ }^{18}$ suggested that a FA/(FA + PY) ratio of $<0.4$ indicates petroleum input, a ratio between 0.4 and 0.5 indicates liquid fossil fuel combustion, while a ratio $>0.5$ indicates grass, wood, or coal combustion. They also reported that the AN/(AN + $\mathrm{PHE})$ ratio of $<0.1$ could be taken as an indication of the petroleum source, while a ratio $>0.1$ indicated combustion as dominant. A ratio of $\mathrm{BaA} /(\mathrm{BaA}+\mathrm{CHR})<0.2$ indicated petrogenic and petroleum sources, $0.2<\mathrm{BaA} /(\mathrm{BaA}+\mathrm{CHR})<0.35$ signaled petroleum combustion, including liquid fossil fuels, vehicles, and crude oil combustion, and $\mathrm{BaA} /(\mathrm{BaA}+\mathrm{CHR})>0.35$ indicated that the source of PAHs were biomass and coal combustion. ${ }^{31}$ Fig. 3 shows AN/(AN + PHE) vs. FA/(FA + PY) and BaA/(BaA $+\mathrm{CHR}) v s$. FA $/(\mathrm{FA}+\mathrm{PY})$. The ratio of $\mathrm{AN} /(\mathrm{AN}+\mathrm{PHE})$ ranged from 0 to $0.45, \mathrm{FA} /(\mathrm{FA}+\mathrm{PY})$ from 0 to 1 , and $\mathrm{BaA} /(\mathrm{BaA}+\mathrm{CHR})$ from 0 to 1 . Over $70 \%$ of the samples had an isomeric ratio that corresponded with a combustion source, while the remaining samples originated from petrogenic sources (Fig. 3a). Fig. 3b also shows a mixed pattern of contamination from pyrogenic and petrogenic sources. All indices above indicated that the sources of PAHs in TCMs were mixed and complicated, deriving from both combustion and petrogenic.

It is noteworthy that, in most root and stem samples (R1-R5), the ratios of FA/(FA + PY), AN/(AN + PHE), and BaA/(BaA + CHR) were all more than $0.5,0.1$, and 0.35 , respectively. These ratios strongly implied sources from grass, wood, or coal combustion, which was consistent with the fact that most roots and stems are dried using combustion gases from burning wood or coal, and that the type of wood has a different influence on the PAH levels produced. ${ }^{32}$

3.5.2. Principal component analysis. In order to enhance the accuracy of source identification, PCA was used to conduct quantitative assessments. When PCA was combined with varimax orthogonal rotation, the variance contribution rate, which explained $83.313 \%$ of the variance, was extracted and five components were identified, as shown in Table 3 (values over 0.53 are highlighted in bold).

Factor 1 explained the total variance of $45.848 \%$ in the data, and was strongly related to ACL, AC, FL, PHE, AN, and FA. PHE and FA have generally been attributed to coal combustion, ${ }^{33,34}$ and ACL, AC and AN have been identified as tracers for PAH compounds emitted by grass or wood combustion. ${ }^{35,36}$ Additionally, this factor was also composed of high molecular weight PAHs with 4-6 rings, such as PY, BbFA, BkFA, IP, DBahA, and BghiP, and are basically known to be derived from the traffic emissions. ${ }^{36,37}$ BghiP has been identified as a tracer of gasoline emissions. IP and BkFA have been found in gasoline vehicle soot, and both gas and diesel engine emissions. ${ }^{38-40}$ Therefore, factor 1 was selected to represent mixed coal, wood combustion, and traffic emissions.

Factor 2 was responsible for $12.736 \%$ of the total variance, and was predominately composed of $\mathrm{BaA}, \mathrm{CHR}$, and $\mathrm{BaP}$, which were indicative of diesel-powered vehicles sources. ${ }^{41}$ This factor could be the vehicle exhaust source category.

Factors 3 and 4 accounted for $9.419 \%$ and $8.830 \%$ of the total variance, respectively, with high loading values of AC and NA 

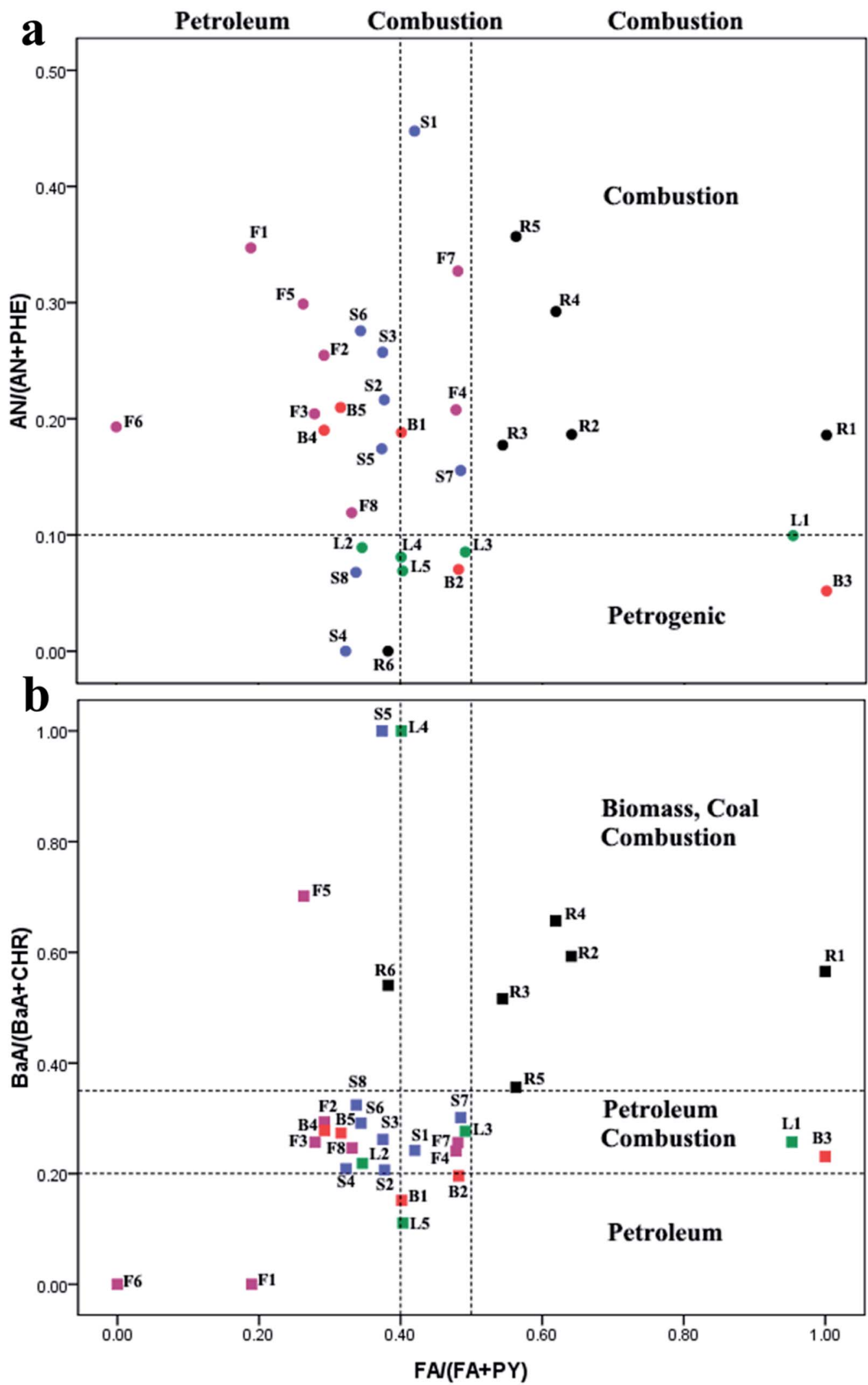

Fig. 3 Cross plots for PAH diagnostic ratios in selected TCMs (a) AN/(AN + PHE) vs. FA/(FA + PY), (b) BaA/(BaA + CHR) vs. FA/(FA + PY). 
Table 3 Rotated component loadings of PAHs in TCMs

\begin{tabular}{|c|c|c|c|c|c|}
\hline Species & \multicolumn{5}{|l|}{ PCA factor loadings } \\
\hline ACL & 0.724 & -0.144 & 0.322 & -0.328 & 0.199 \\
\hline $\mathrm{AC}$ & 0.525 & -0.122 & 0.600 & 0.303 & -0.165 \\
\hline FL & 0.618 & -0.433 & 0.421 & -0.338 & -0.158 \\
\hline FA & 0.943 & -0.158 & -0.159 & -0.070 & 0.044 \\
\hline PY & 0.707 & -0.389 & 0.110 & -0.236 & -0.391 \\
\hline $\mathrm{BaA}$ & 0.396 & 0.658 & 0.410 & -0.262 & 0.024 \\
\hline CHR & 0.167 & 0.682 & 0.278 & -0.321 & 0.391 \\
\hline BbFA & 0.655 & 0.520 & -0.089 & 0.094 & -0.302 \\
\hline BkFA & 0.625 & 0.161 & -0.182 & -0.008 & -0.227 \\
\hline Contribution (\%) & 45.848 & 12.736 & 9.419 & 8.830 & 6.480 \\
\hline Possible source & $\begin{array}{l}\text { Combustion (coal/wood) } \\
\text { traffic emission }\end{array}$ & Vehicle exhaust & Petrogenic source & Petrogenic source & Coal combustion \\
\hline
\end{tabular}

respectively, both suggesting petrogenic source. ${ }^{29,42}$ Factor 5 contributed $6.480 \%$ to the total variance, containing only one highly loaded component, DBahA, which might be related to coal combustion. ${ }^{43}$

Generally, the results of PCA analyses were in concordance with the evidence from the diagnostic ratios of PAHs, which revealed a mixture of pyrogenic and petrogenic-derived PAHs. Vehicular emissions, petrogenic sources, wood and coal combustion may be responsible for the PAHs found in TCMs in the present study.

\section{Conclusion}

Different extraction methods (ultrasonic extraction, homogenization extraction, and oscillation extraction) were adopted according to the different matrices contained in the different medicinal parts of TCMS, followed by silica gel cleanup and HPLC-UV-FLD detection for the analysis of PAHs in TCMs. The proposed method was proved to be easy, cheap, effective, and accurate, and was successfully applied to determine PAHs in 32 TCMs of different types, including roots and stems, leaves, flowers, seeds, and fruits. The contamination of PAHs in TCMs seemed especially serious in leaves, roots, and stems. All 32 TCMs were contaminated with some PAHs, but with a large variability concerning PAHs distribution in different samples or medicinal parts. PAHs diagnostic ratios and PCA analysis indicated that PAHs in TCMs were from both pyrogenic and petrogenic sources. Wood or coal combustion could be responsible for the majority of PAHs found in roots and stems. The consumption of TCMs, especially by patients suffering chronic diseases, may be a long-term process and, thus, needs strict quality control while no legislation to regulate the contamination of TCMs with PAHs. The method presented in this study could be promoted as a useful tool for the quantification of PAHs contents in TCMs. The data obtained could also provide valuable information for further studies of the maximum residue limits and potential health risk assessment.

\section{Acknowledgements}

We thank Lianju Ma (College of Pharmacy, Chongqing Medical University) for the assistance in HPLC-FLD analysis.

\section{References}

1 Q. Yang, H. Chen and B. Li, PLoS One, 2015, 10, e0118141.

2 T. H. Kao, S. Chen, C. J. Chen, C. W. Huang and B. H. Chen, J. Agric. Food Chem., 2012, 60, 1380-1389.

3 T. Wenzl, R. Simon, E. Anklam and J. Kleiner, TrAC, Trends Anal. Chem., 2006, 25, 716-725.

4 R. Liu, Y. Dai and L. Sun, PLoS One, 2015, 10, e0120369.

5 A. Ishizaki, K. Sito and H. Kataoka, Anal. Methods, 2011, 3, 299-305.

6 I. Hosbach, G. Neeb, S. Hager, S. Kirchhoff and B. Kirschbaum, Anaesthesia, 2003, 58, 282-283.

7 L. Veiga, H. Amorim, J. Moraes, M. Silva, R. Raices and S. Quiterio, Food Chem., 2014, 152, 612-618.

8 M. Fernández, S. Clavijo, R. Forteza and V. Cerdà, Talanta, 2015, 138, 190-195.

9 Z. Cui, N. Ge, A. Zhang, Y. Liu, J. Zhang and Y. Cao, Anal. Bioanal. Chem., 2015, 407, 1989-1997.

10 L. Yu, Y. Cao, J. Zhang, Z. Cui and H. Sun, Food Addit. Contam., Part A, 2012, 29, 1800-1809.

11 K. Dost and C. İdeli, Food Chem., 2012, 133, 193-199. 
12 H. Yin, Q. Tan, Y. Chen, G. Lv and X. Hou, Microchem. J., 2011, 97, 138-143.

13 D. Lin, Y. Tu and L. Zhu, Food Chem. Toxicol., 2005, 43, 4148.

14 M. V. Russo, P. Avino, L. Perugini and I. Notardonato, RSC Adv., 2016, 6, 13920-13927.

15 M. Surma, A. Sadowska-Rociek and E. Cieślik, Eur. Food Res. Technol., 2014, 238, 1029-1036.

16 R. M. Harrison, D. Smith and L. Luhana, Environ. Sci. Technol., 1996, 30, 825-832.

17 C. Bourotte, M.-C. Forti, S. Taniguchi, M. C. Bícego and P. A. Lotufo, Atmos. Environ., 2005, 39, 3799-3811.

18 M. B. Yunker, R. W. Macdonald, R. Vingarzan, R. H. Mitchell, D. Goyette and S. Sylvestre, Org. Geochem., 2002, 33, 489-515.

19 I. Primpas and M. Karydis, Environ. Monit. Assess., 2011, 178, 257-269.

20 R. K. Larsen and J. E. Baker, Environ. Sci. Technol., 2003, 37, 1873-1881.

21 B. Kumar, V. Verma, R. Gaur, S. Kumar, C. Sharma and A. Akolkar, Adv. Appl. Sci. Res., 2014, 5, 201-209.

22 M. W. Ashraf and A. Salam, Bull. Environ. Contam. Toxicol., 2012, 88, 543-547.

23 S. A. Tfouni, R. M. Machado, M. C. Camargo, S. H. Vitorino, E. Vicente and M. C. F. Toledo, Food Chem., 2007, 101, 334338.

24 S. Singh and A. Vashishth, Environ. Monit. Assess., 2011, 177, 35-38.

25 H. Krajian and A. Odeh, Toxicol. Environ. Chem., 2013, 95, 942-953.

26 M. Ciecierska and M. Obiedzinski, Herba Pol., 2009, 55, 1824.

27 K. Srogi, Environ. Chem. Lett., 2007, 5, 169-195.
28 N. R. Bishnoi, U. Mehta, U. Sain and G. Pandit, Environ. Monit. Assess., 2005, 107, 399-406.

29 Z. Wang, M. Fingas, Y. Shu, L. Sigouin, M. Landriault, P. Lambert, R. Turpin, P. Campagna and J. Mullin, Environ. Sci. Technol., 1999, 33, 3100-3109.

30 Z. Ouyang, L. Gao, X. Chen, S. Yao and S. Deng, RSC Adv., 2016, 6, 71441-71449.

31 A. Dvorská, G. Lammel and J. Klánová, Atmos. Environ., 2011, 45, 420-427.

32 B. K. Larsson, Z. Lebensm.-Unters. Forsch., 1982, 174, 101107.

33 U. M. Sofowote, B. E. McCarry and C. H. Marvin, Environ. Sci. Technol., 2008, 42, 6007-6014.

34 G.-L. Shi, Y.-Z. Tian, C.-S. Guo, Y.-C. Feng, J. Xu and Y. Zhang, J. Soils Sediments, 2012, 12, 1154-1163.

35 N. R. Khalili, P. A. Scheff and T. M. Holsen, Atmos. Environ., 1995, 29, 533-542.

36 Y. Zhang, C.-S. Guo, J. Xu, Y.-Z. Tian, G.-L. Shi and Y.-C. Feng, Water Res., 2012, 46, 3065-3073.

37 H.-Y. Chen, Y.-G. Teng and J.-S. Wang, Sci. Total Environ., 2012, 414, 293-300.

38 Y. Liu, L. Chen, Q.-H. Huang, W.-Y. Li, Y.-J. Tang and J.-F. Zhao, Sci. Total Environ., 2009, 407, 2931-2938.

39 M. P. Zakaria, H. Takada, S. Tsutsumi, K. Ohno, J. Yamada, E. Kouno and H. Kumata, Environ. Sci. Technol., 2002, 36, 1907-1918.

40 R. Boonyatumanond, M. Murakami, G. Wattayakorn, A. Togo and H. Takada, Sci. Total Environ., 2007, 384, 420432.

41 P. Masclet, G. Mouvier and K. Nikolaou, Atmos. Environ., 1967, 1986(20), 439-446.

42 H. Soclo, P. Garrigues and M. Ewald, Mar. Pollut. Bull., 2000, 40, 387-396.

43 T. T. Dong and B.-K. Lee, Chemosphere, 2009, 74, 1245-1253. 